\title{
Cell- and region-specific miR30-based gene knock-down with temporal control in the rat brain
}

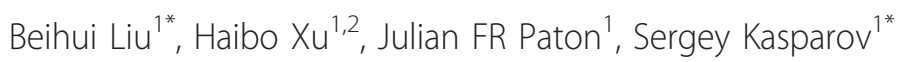

\begin{abstract}
Background: RNA interference (RNAi) emerges as a powerful tool to induce loss-of-function phenotypes. In the context of the brain, gene manipulation is best targeted to specific subsets of cells in order to achieve a physiologically relevant outcome. Polymerase II-based viral expression systems can be used to cell-specifically express constructs incorporating flanking and loop sequences from endogenous microRNA (miRNA), which directs the designed hairpins into the endogenous gene silencing machinery. While many studies have documented noncell-selective gene knock-down in the brain, it has not been tested whether different cell types or different areas of the central nervous system (CNS) are equally amenable to this approach. We have evaluated this issue using a tetracycline (Tet)-controllable and cell-specific miRNA 30 (miR30)-based short hairpin (shRNA) interference system.

Results: To achieve targeted expression two cell type-specific promoters were used; the enhanced compact glial fibrillary acidic protein $\left(G G_{A} A B C_{1} D\right)$ promoter and the enhanced human synapsin-1 (SYN) promoter. Powerful luciferase (Luc) and the neuronal isoform of nitric oxide synthase (nNOS) gene knock-down were achieved both in vitro and in vivo. Administration of doxycycline (Dox) abrogated gene silencing. However, the efficacy of gene knock-down in both neurones and astrocytes in the hippocampus (HIP) was lower than that in the dorsal vagal complex of the brainstem (DVC). This was not due to regional differences in the expression of the the key enzymes involved in miRNA processing.

Conclusions: The results from the presented experiments demonstrated that selective gene knock-down in subsets of brain cells is achievable. However, there are some presently unknown regional factors which affect either the processing of miRNA-based cassettes or their potency for gene silencing.
\end{abstract}

\section{Background}

RNAi is a conserved regulatory process that mediates sequence-specific post-transcriptional gene silencing in a variety of species $[1,2]$. The RNAi technique has become a potent experimental tool for functional analysis of genes and is believed to have significant therapeutic potential [3-6]. In the context of the brain, where numerous cell types with different functions are intermixed, gene knock-down targeted to a cellular specified phenotype in most cases would be preferable, in order to achieve a physiologically or medically relevant outcome $[7,8]$. Within vector-based RNAi systems, miRNA-

\footnotetext{
* Correspondence: bh.liu@bristol.ac.uk; Sergey.Kasparov@bristol.ac.uk 'Department of Physiology and Pharmacology, Bristol Heart Institute, School of Medical Sciences, University of Bristol, UK

Full list of author information is available at the end of the article
}

based cassettes, in which shRNA is embedded in a precursor miRNA context, are particularly attractive [9-12]. Endogenous miRNAs are transcribed predominantly by polymerase II (Pol II) promoters as long primary miRNAs transcripts (pri-miRNAs) that are subsequently processed into precursor miRNAs (pre-miRNAs) and eventually incorporated into the RNA-induced silencing complex (RISC) to mediate silencing of the target gene ([9]). Vectors can be made to mimic naturally occurring miRNAs whereby a designed pre-miRNA is embedded in an authentic miRNA context enabling its entry into the endogenous miRNA/RNAi pathway $[9,11,13,14]$. The value of this approach was demonstrated by Stegmeier et al who achieved an effective knock-down with only a single viral genome per cell [15]. This is an important feature given that it is hard to achieve high
C Biomed Central

(c) 2010 Liu et al; licensee BioMed Central Ltd. This is an Open Access article distributed under the terms of the Creative Commons Attribution License (http://creativecommons.org/licenses/by/2.0), which permits unrestricted use, distribution, and reproduction in any medium, provided the original work is properly cited. 
copy numbers in a single cell in the brain. Moreover, large libraries of pre-designed constructs of this type are commercially available from OpenBiosystems and Invitrogen. Typically, in these constructs the miRNA-like motifs are fused to the end of a sequence encoding a fluorescent protein, giving rise to a transcript which encodes both the pre-miRNA and the reporter gene. As this is a Pol II- driven system it can be controlled by a tetracycline (or its analogue Dox)-responsive promoter element (Tre) as used in many viral systems [16-18]. If expression of the transactivator such as the Tet-off transactivator (tTA) is cell type specific, this would allow targeting gene knock-down to a specific cell type. As the same gene frequently co-exists in both neurones and astrocytes in the brain and may have different or even opposite functions, cell-selective gene manipulation has obvious advantages. In addition, for most experimental and potential clinical applications, viral gene knock-down might be most advantageous if targeted to a selected brain region(s). It is usually assumed that the mechanisms of miRNA-mediated gene knock-down are ubiquitous and operate constitutively in various tissues [3,19-21]. However, concerning the brain, it has not yet been tested whether the same gene knock-down cassette is equally effective in different areas of the central nervous system (CNS).

There are two main objectives for this study: Firstly, we asked whether cell-specific and Dox-controllable gene knock-down can be achieved in both astrocytes and neurones. Secondly, we compared the efficacy of gene knock-down in two very different areas of the CNS, an evolutionary "old" part of the brain within the medulla oblongata involved with the control of various autonomic functions and a much "younger" part of the brain, the hippocampus (HIP). To this end, we constructed a binary Dox-controllable and cell-specific miR30-based RNAi system to express shRNAs targeting a reporter gene for Luc and an endogenous gene for nNOS. Luc was chosen as it allowed a quantifiable assessment of the efficacy of gene knock-down at the protein level. Furthermore, Luc quantification is reliable and technically straightforward. nNOS was chosen as a typical neuronal protein which is expressed in both areas of the brain we chose to study at moderate levels [22-25]. In these constructs gene targeting sequences were embedded in the precursor miRNA context derived from miR30, one of the most well-studied miRNA in mammals. This construct was fused to the 3' end of the GFP sequence and placed under control of the Tre promoter within a lentiviral vector (LVV) backbone. To drive its expression in a cell-specific manner it was co-applied with the other LVV expressing Tet-off transactivator tTA using either $\mathrm{GfaABC}_{1} \mathrm{D}$ promoter for astrocytes or SYN promoter for neurones, both promoters were transcriptionally enhanced using previously published approaches [26]. This yielded in a binary system which was both, cell-specific and Doxcontrollable.

We demonstrate that significant knock-down can be achieved in a cell-selective and Dox-controllable manner for both an exogenous and endogenous targeted gene. However, there are major regional differences in the efficacy of the same gene knock-down cassette, which cannot be explained by the differential expression of the main known proteins involved in miRNA processing.

\section{Methods}

\section{LVVs used in this study}

Six LVVs were constructed for this study (Figure 1). (1) LV-Tretight-GFP-miR30-shRNA/Luc where the GFP sequence is followed by a miR30-embedded anti-Luc hairpin under the control of the Tretight promoter; (2) LV-Tretight-GFP-miR30-shRNA/nNOS, in which the GFP sequence is followed by a miR30-embedded antinNOS hairpin under the control of the Tretight promoter; (3) LV-GfaABC ${ }_{1} \mathrm{D}$-Luc where the Luc gene is expressed by the astrocytic $\mathrm{GfaABC}_{1} \mathrm{D}$ promoter; (4) LV-SYN-Luc, in which the Luc gene is expressed by the neuronal SYN promoter; (5) LV-mCMV/SYN-tTA where the tTA gene expression is under the control of the enhanced SYN promoter by the bidirectional transcriptional amplification strategy [26]; and (6) LV$\mathrm{mCMV} / \mathrm{GfaABC}_{1} \mathrm{D}$-tTA in which the tTA gene expression is under the control of the enhanced $G_{f a A B C} D$ promoter by the bidirectional transcriptionally amplification strategy. The Luc targeting shRNA sequence was provided by Dr. M.Z. Li (Howard Hughes Medical Institute, Boston) and the nNOS targeting sequence was designed by using the RNAi design algorithm at http:// katahdin.cshl.org/siRNA/RNAi.cgi?type=shRNA and kindly provided by Dr. Luna Benvenisti (Zarom Teva Pharmaceutical Industries, USA). LV-GfaABC ${ }_{1}$ D-Luc and LV-SYN-Luc served as sources of Luc in either an astrocyte- or neurone-specific manner. Additionally LVSYN-WPRE and LV-GfaABC1D-WPRE (unpublished constructs in our lab, not illustrated) worked as control vectors to balance the total load of viral particles into cells or animal tissues.

\section{Plasmid construction}

All the LVV plasmids were based on the improved lentiviral shuttle vector pTYF-SW-Linker backbone using standard cloning procedures [27]. To generate the LVTretight-GFP-miR30-shRNA/Luc shuttle vector, we excised the Tretight fragment containing the modified Tet-responsive promoter from pTRE-Tight-DsRed2 (Clontech) and inserted it into the pTYF-SW Linker and cloned, into the obtained vector, PCR product of 

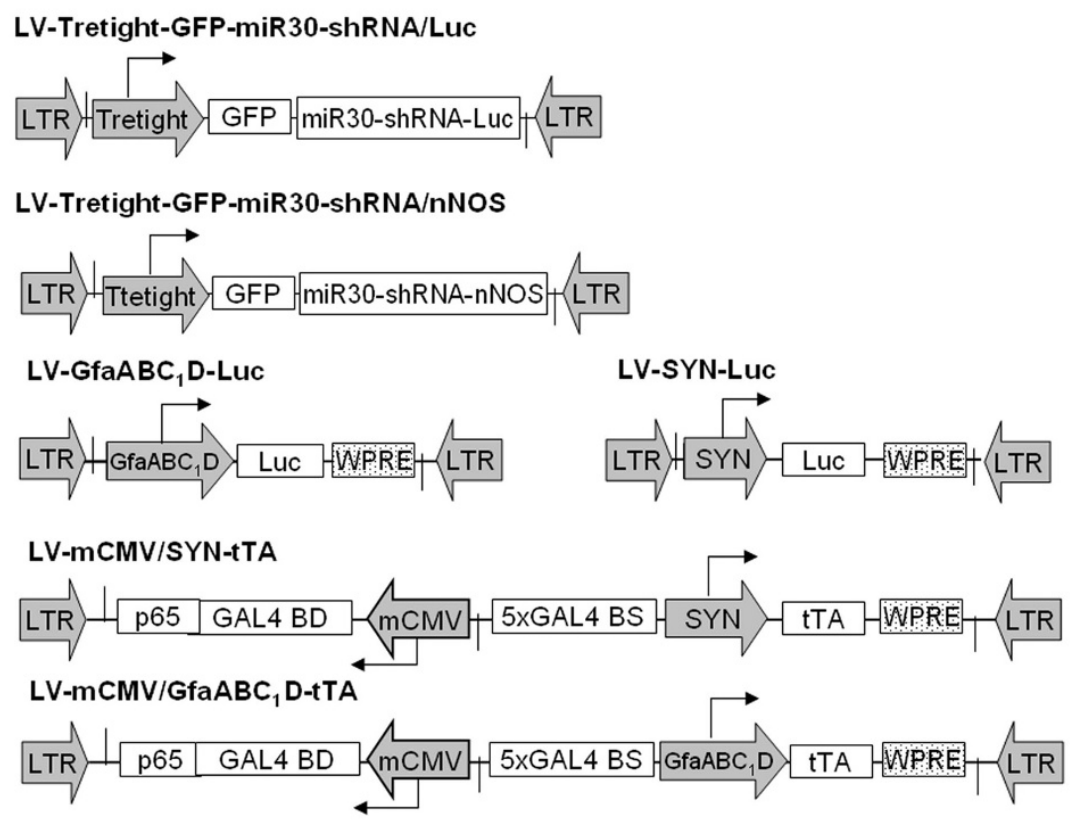

Figure 1 Schematic representation of lentiviral vectors used in the current study. LTR, lentiviral long terminal repeats; Tretight, a modified tetracycline and Dox-responsive promoter derived from pTRE-tight (Clontech); GFP, green fluorescence protein; miR30-shRNA/Luc, miR30-based shRNA targeting firefly Luc gene; miR30-shRNA/nNOS, miR30-based shRNA targeting rat neuronal nitric oxide synthase gene; Luc, firefly Luc gene; GfaABC 1 D, a compact glial fibrillary acidic protein promoter (690 bp); SYN, human synapsin 1 promoter (470 bp); mCMV, minimal CMV core promoter (65 bp); GAL4BDp65, a chimeric transactivator consisting of a part of the transactivation domain of the murine NF- $\kappa$ Bp65 protein fused to the DNA binding domain of GAL4 protein from yeast; WPRE, woodchuck hepatitis post-transcriptional regulatory element.

GFP-miR30-shRNA/Luc cassette from pPRIME-CMVGFP-FF3 (kindly provided by F. Stegmeier, Harvard Medical School) downstream of Tretight promoter. To construct the LV-Tretight-GFP-miR30-shRNA/nNOS shuttle vector, we replaced the Luc shRNA sequence in the LV-Tretight-GFP-miR30-shRNA/Luc shuttle vector with the nNOS shRNA. We obtained LV-Gfa $\mathrm{ABC}_{1} \mathrm{D}$ Luc and LV-SYN-Luc shuttle vectors by replacing EGFP in $\mathrm{pTYF}-1 \times \mathrm{GfaABC}_{1} \mathrm{D}$-EGFP and $\mathrm{PTYF}-1 \times \mathrm{SYN}$-EGFP [26] with the Luc cDNA from the pGL3-Basic vector (Promega) respectively. LV-mCMV/GfaABC ${ }_{1} \mathrm{D}-\mathrm{tTA}$ and LV-mCMV/SYN-tTA shuttle vectors were both produced by replacing EGFP in pTYF-mCMV/SYN-EGFP and pTYF-mCMV/GfaABC ${ }_{1}$ D-EGFP [26] with the tTA cDNA from the LV-1×SYN-tTA shuttle vector [7].

\section{LVV production}

The LVV system used in this study is derived from HIV-1 and pseudotyped with the vesicular stomatitis virus coat [28]. LVV stocks were produced by transient co-transfection of the shuttle plasmids, the packaging vector $\mathrm{pNHP}$, and the envelope plasmid pHEF-VSVG in HEK293FT cells. Viral concentration and titration were carried out as described earlier [28].

\section{Cell culture and in vitro LVV vector transduction}

The in vitro transduction experiments were carried out in a neurone-derived rat pheochromocytoma PC12 cell line and a $1321 \mathrm{~N} 1$ glial cell line from human brain astrocytoma. PC12 cells were grown in Dulbecco's modified Eagle's medium (DMEM) supplemented with 10\% heat-inactivated fetal bovine serum (FBS) and 5\% horse serum. $1321 \mathrm{~N} 1$ cells were cultured in DMEM supplemented with $10 \%$ heat-inactivated FBS. The cells were split and plated in 24-well plates at a cell density of $5 \times$ $10^{4}$ per well with $0.5 \mathrm{ml}$ culture medium. After $24 \mathrm{~h}$, cells were transduced overnight with appropriate LVVs in the presence of polybrene $(8 \mu \mathrm{g} / \mathrm{ml})$. The ratio for three vectors in all cotransfections was fixed to $1: 1: 1$ and the total viral MOI per well was fixed to 5. Cells were then washed in phosphate-buffered saline (PBS) and cultured in full media for a further $48 \mathrm{~h}$. For each virus combination, 3 wells were transduced. At the end of the incubation, cells were washed and permeabilized with $100 \mu \mathrm{l}$ of reporter cell lysis buffer (Promega) for Luc activity assay or $100 \mu \mathrm{l}$ of radioimmunoprecipitation (RIPA) buffer (50 mM Tris, 1\% NP-40, 1\% Sodium Deoxycholate, 0.1\% SDS, $150 \mathrm{mM} \mathrm{NaCl}, 1 \mathrm{mM}$ EDTA, $\mathrm{pH}$ 7.5) plus a protease inhibitor cocktail (Sigma) for 
western blot analysis respectively. The lysed samples can be kept at $-80^{\circ} \mathrm{C}$ until processing.

\section{In vivo stereotaxic injections}

All experiments were carried out in accordance with the Animals Scientific Procedures Act 1986. The animals were housed individually, allowed normal rat chow and drinking water ad libitum, and kept on a 12-hour light/12-hour dark cycle. Briefly, adult male Wistar rats (200 250 g) were anaesthetized with ketamine and medetomidine intramuscularly and their heads mounted securely in a stereotaxic frame. LVV were microinjected stereotaxically into either the DVC including the hypoglossal motor nucleus as before [8] or HIP at the following coordinates: anterior, $-4.4 \mathrm{~mm}$; lateral, $+3.2 \mathrm{~mm}$; ventral, $-2.5 \mathrm{~mm}$ from the surface of the dura as described in [29]. A total of six microinjections of viral vectors were made bilaterally for both DVC and HIP injections. In cases where three viral vectors had to be co-injected, their ratio was fixed to 1:1:1 and the total dose was $6 \times 10^{6}$ infection unit (iu) per rat. In cases where two viral vectors were co-injected, their ratio was fixed to 1:4 and the total dose was also $6 \times 10^{6}$ iu per rat. The injection rate was $0.5 \mu \mathrm{l} / \mathrm{min}$ and the injection needle was allowed to remain in situ for $5 \mathrm{~min}$ before being slowly retracted at the end of each injection. Dox was administered at a concentration of $2 \mathrm{mg} / \mathrm{ml}$ supplemented with $5 \%$ sucrose in the animals' drinking water as required. Seven days postinjection, rats were terminally anaesthetized (sodium pentobarbital, $100 \mathrm{mg} / \mathrm{kg}$ intramuscularly) and perfused through the heart with $0.1 \mathrm{M}$ PBS (pH 7.4). For Luc activity assays, the brain tissue samples were removed and stored at $-80^{\circ} \mathrm{C}$ until processing. After adding PBS buffer (100 $\mu \mathrm{l}$ PBS per $50 \mathrm{mg}$ tissue), each sample was homogenized by sonication for $10 \mathrm{sec}$ on ice, and then centrifuged at $13000 \mathrm{rpm}$ at $4^{\circ} \mathrm{C}$. In total, $10 \mu \mathrm{l}$ of the supernatant at room temperature was used for the Luc activity assay. For nNOS western blot analysis, the brain tissue samples were removed and immediately homogenized with a manual homogenizer in RIPA buffer containing protease inhibitor cocktail. Total protein extracts were then kept at $-80^{\circ} \mathrm{C}$ until further processing.

\section{Luciferase assay}

Luciferase assay was performed with a luciferase assay kit (Promega) in a single-tube luminometer as described [29]. The results are expressed in relative light units (RLU) per well of a 24-well plate for in vitro experiments or per rat region for in vivo experiments. Data are expressed as the mean \pm standard deviation.

\section{Western blot analysis}

The nNOS western blot analysis was carried out as previously described [30]. Briefly, total protein was extracted from homogenized samples, followed by quantification with a BCA protein assay kit (Pierce). $20 \mu \mathrm{g}$ of total protein per lane were separated on NuPAGE 4-12\% Bis-Tris gels (Invitrogen) and transferred to PVDF membranes (Millipore). The membranes were blocked in 5\% non-fat dry milk (NFDM) in Trisbuffered saline with $0.1 \%$ tween-20 (TBST) for $45 \mathrm{~min}$, and incubated with polyclonal rabbit anti-nNOS antibody (Zymed) at 1:5000 in 3\% NFDM-TBST or monoclonal anti-beta-actin antibody (Sigma) at 1:5000 in 1\% BSA-TBST overnight. Following incubation with polyclonal swine anti-rabbit immunoglobulins/HRP (Dako) at 1:5000 in 3\% NFDM-TBST or polyclonal rabbit antimouse immunoglobulins/HRP (Dako) at 1:10000 in 1\% BSA-TBST for $90 \mathrm{~min}$, the immunoreactions were detected with an Immun-Star Western chemiluminescent kit (Bio-Rad) and Amersham high performance autoradiography film (GE Healthcare). Scion Image software (Scion Corporation) was used to quantitatively compare the relative blot intensities.

\section{Northern blot analyses}

Total RNA was isolated from rat tissues seven days post-injection using mirVana Isolation Kit (Ambion). Fifteen micrograms total RNA were fractionated on a $15 \%$ denaturing polyacrylamide gel and blotted onto Hybond-XL membrane (Amersham). RNA was immobilized by UV crosslinking and baking for 1 hour at $80^{\circ} \mathrm{C}$. Hybridization was carried out at $42^{\circ} \mathrm{C}$ using UltraHybOligo Hybridization buffer (Ambion). Probes were labeled with ${ }^{32} \mathrm{P}$ using T4 polynucleotide kinase (New England Biolabs). Membranes were washed twice in $2 \times S S C, 0.1 \%$ SDS and $0.2 x S S C, 0.1 \%$ SDS at $37^{\circ} \mathrm{C}$ and exposed to film. Scion Image software (Scion Corporation) was used to quantitatively compare the relative blots intensities.

\section{Real time RT-PCR}

Total RNA was isolated from cell lines and rats using RNAqueous-Micro kit (Ambion) and treated with DNase I. RNA purity was verified by performing PCR on samples not treated with reverse transcriptase. Realtime RT-PCRs were carried out using a DNA Engine Opticon 2 system (MJ Research) and the QuantiTect SYBR Green RT-PCR kit (Qiagen), as described [31]. Expression of target genes was assessed in relation to a housekeeping gene $(\beta$-actin) using the comparative Pfaffl

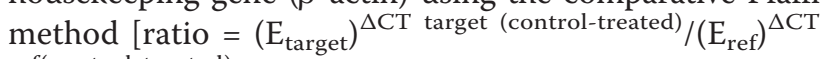
ref(control-treated) ] in each sample [32]. Fold differences against DVC were calculated $(\mathrm{n}=5)$. PCR primers for rat Dicer, Argonaute proteins (Ago), Digeorge syndrome critical region gene 8 (DGCR8), Exportin 5 and Drosha were designed according to sequence information provided by NCBI with the accession numbers: XM_001069041, XM_001058231, NM_001105865, 
NM_001108789 and NM_001107655 respectively. Among the above, XM_001069041 for Dicer and XM_001058231 for Ago are predicted sequences by automated computational analysis using gene prediction method: GNOMON, supported by mRNA and EST evidence. Primer sequences are detailed in the Additional file 1.

\section{Results}

\section{Combinations of vectors used in this study}

Ten different viral combinations were used here. To simplify the presentation, we used abbreviations. Combinations and abbreviations are presented in Table 1.

\section{Analyses of the functions of miR30-shRNA/Luc in vitro}

First, the effect of miR30-shRNA/Luc was assessed in cell lines. PC12 cells were co-transduced with viral combinations of LVVs-miRLuc-control1 and LVVs-miRLucneurone while $1321 \mathrm{~N} 1$ cells were co-transduced with LVVs-miRLuc-control2 and LVVs-miRLuc-glia. In the absence of Dox, Luc expression from LVVs-miRLucneurone [Figure $2(a)$, group $B$ ] was significantly knocked down by $80 \%$ as compared to that from LVVs-miRLuccontrol1 [Figure 2(a), group A] [5.37 $\times 10^{5} \pm 9.25 \times 10^{4}$ vs $2.8 \times 10^{6} \pm 2.67 \times 10^{5}$ RLU per well; $\left.P<0.01\right]$ in PC12 cells. Similarly, in $1321 N 1$ cells, Luc expression from LVVs-miRLuc-glia [Figure 2(b), group B'] was significantly reduced by $90 \%$ as compared to that from LVVs-miRLuc-control1 [Figure 2(b), group A'] $(4.79 \times$ $10^{4} \pm 6.54 \times 10^{3}$ vs $4.85 \times 10^{5} \pm 1.81 \times 10^{4} \mathrm{RLU}$ per well; $P<0.01)$. The Luc knock-down was completely prevented in the presence of Dox in both PC12 and 1321N1 cells [Figure 2(a), group C vs B and Figure 2(b), group C' vs B']. The inhibitory effect of Dox disappeared after three days of Dox withdrawal [Figure 2(a), group D vs $C$ and Figure $2(b)$, group D' vs $\left.C^{\prime}\right]$. These results demonstrate that bidirectional transcriptionally amplified $S Y N$ and $G_{a A B C} D$ promoters provide a sufficient level of tTA to activate the Tretight promoter which then drives the synthesis of GFP-miR30-shRNA/Luc transcript to induce substantial Luc knock-down.

Of note, the astrocytic system LVVs-miRLuc-glia was more effective than the neuronal system, LVVs-miRLucneurone. We therefore tested whether this could reflect a higher efficacy of the amplification strategy when applied to the $\mathrm{GfaABC}_{1} \mathrm{D}$ as compared to $\mathrm{SYN}$ promoter. To this end, we performed a real time PCR analysis of tTA expression level from $\mathrm{LV}$-mCMV/GfaABC ${ }_{1} \mathrm{D}$-tTA in

Table 1 Viral combinations used in this study and their functions

\begin{tabular}{|c|c|c|}
\hline Abbreviation & Vector combination & Function \\
\hline $\begin{array}{l}\text { LVVs-miRLuc- } \\
\text { neurone }\end{array}$ & $\begin{array}{l}\text { LV-SYN-Luc } \\
+ \text { LV-Tretight-GFP-miR30-shRNA/Luc } \\
+ \text { LV-mCMV/SYN-tTA }\end{array}$ & $\begin{array}{l}\text { Neurone-specific Luc knock-down system. Tet-off transactivator tTA is expressed from } \\
\text { LV-mCMV/SYN-tTA which contains the bidirectional amplified SYN promoter to ensure } \\
\text { high level of tTA. tTA binds to Tretight promoter in LV-Tretight-GFP-miR30-shRNA/Luc } \\
\text { and activates the expression of shRNA/Luc. }\end{array}$ \\
\hline LVVs-miRLuc-glia & $\begin{array}{l}\text { LV-GfaABC } 1 \text { D-Luc } \\
+ \text { LV-Tretight-GFP-miR30-shRNA/Luc } \\
+ \text { +LV-mCMV/GfaABC1D-tTA }\end{array}$ & $\begin{array}{l}\text { Astrocyte-specific Luc knock-down system. Transactivator tTA is expressed from LV- } \\
\text { mCMV/GFABC1D-tTA which contains the bidirectional amplified GfaABC1 D promoter } \\
\text { to ensure high level of tTA. tTA binds to Tretight promoter in LV-Tretight-GFP-miR30- } \\
\text { shRNA/Luc and activates the expression of shRNA/Luc. }\end{array}$ \\
\hline $\begin{array}{l}\text { LVVs-miRLuc- } \\
\text { control1 }\end{array}$ & $\begin{array}{l}\text { LV-SYN-LuC } \\
+ \text { LV-Tretight-GFP-miR30-shRNA/Luc } \\
+ \text { LV-SYN-WPRE }\end{array}$ & $\begin{array}{l}\text { Control combination used in Luc knock-down experiments for the neurone-specific } \\
\text { system. Since there is no tTA expression in this combination, shRNA/Luc will not be } \\
\text { expressed. So this combination marked the background expression level of Luc driven } \\
\text { by SYN promoter. }\end{array}$ \\
\hline $\begin{array}{l}\text { LVVs-miRLuc- } \\
\text { control2 }\end{array}$ & $\begin{array}{l}\text { LV-GfaABC } 1 \text { D-Luc } \\
+ \text { LV-Tretight-GFP-miR30-shRNA/Luc } \\
+ \text { LV-GfaABC1D-WPRE }\end{array}$ & $\begin{array}{l}\text { Control combination used in Luc knock-down experiments for the astrocyte-specific } \\
\text { system. Similar as LWs-miRLuc-control 1, there is no tTA expression in this } \\
\text { combination; shRNA/Luc will not be expressed. Therefore this combination marked the } \\
\text { background expression level of Luc driven by GfaABC1 D promoter. }\end{array}$ \\
\hline $\begin{array}{l}\text { LVVs-miRnNOS- } \\
\text { neurone }\end{array}$ & $\begin{array}{l}\text { LV-Tretight-GFP-miR30-shRNA/nNOS } \\
\quad+\text { LV-mCMV/SYN-tTA }\end{array}$ & $\begin{array}{l}\text { Neurone-specific nNOS knock-down system. Mechanism is similar as LWs-miRLuc- } \\
\text { neurone. }\end{array}$ \\
\hline LVVs-miRnNOS-glia & $\begin{array}{l}\text { LV-Tretight-GFP-miR30-shRNA/nNOS } \\
\quad+\text { LV-mCMV/GfaABC1D-tTA }\end{array}$ & Astrocyte-specific Luc knock-down system. Mechanism is similar as LWs-miRLuc-glia. \\
\hline $\begin{array}{l}\text { LVVs-miRnNOS- } \\
\text { control1 }\end{array}$ & $\begin{array}{l}\text { LV-Tretight-GFP-miR30-shRNA/nNOS } \\
+ \text { LV-SYN-WPRE }\end{array}$ & $\begin{array}{l}\text { Control combination used in nNOS knockdown experiments for the neurone-specific } \\
\text { system. Mechanism is similar as LWs-miRLuc-control1. }\end{array}$ \\
\hline $\begin{array}{l}\text { LVVs-miRnNOS- } \\
\text { control2 }\end{array}$ & $\begin{array}{l}\text { LV-Tretight-GFP-miR30-shRNA/nNOS } \\
\quad+\text { LV-GfaABC } 1 \text { D-WPRE }\end{array}$ & $\begin{array}{l}\text { Control combination used in nNOS knockdown experiments for the astrocyte-specific } \\
\text { system. Mechanism is similar as LWs-miRLuc-control2. }\end{array}$ \\
\hline $\begin{array}{l}\text { LVVs-miRnNOS- } \\
\text { negative control1 }\end{array}$ & $\begin{array}{l}\text { LV-Tretight-GFP-miR30-shRNA/Luc } \\
\quad+\text { LV-mCMV/SYN-tTA }\end{array}$ & $\begin{array}{l}\text { Negative control combination used in nNOS knock-down experiments for the } \\
\text { neurone-specific system. Instead of producing shRNA/nNOS it produces shRNA/Luc } \\
\text { which shouldn't work on nNOS knockdown. }\end{array}$ \\
\hline $\begin{array}{l}\text { LVVs-miRnNOS- } \\
\text { negative control2 }\end{array}$ & $\begin{array}{l}\text { LV-Tretight-GFP-miR30-shRNA/Luc } \\
\quad+\text { LV-mCMV/GfaABC1D-tTA }\end{array}$ & $\begin{array}{l}\text { Negative control combination used in nNOS knock-down experiments for the } \\
\text { astrocyte-specific system. Instead of producing shRNA/nNOS it produces shRNA/Luc } \\
\text { which shouldn't work on nNOS knockdown. }\end{array}$ \\
\hline
\end{tabular}




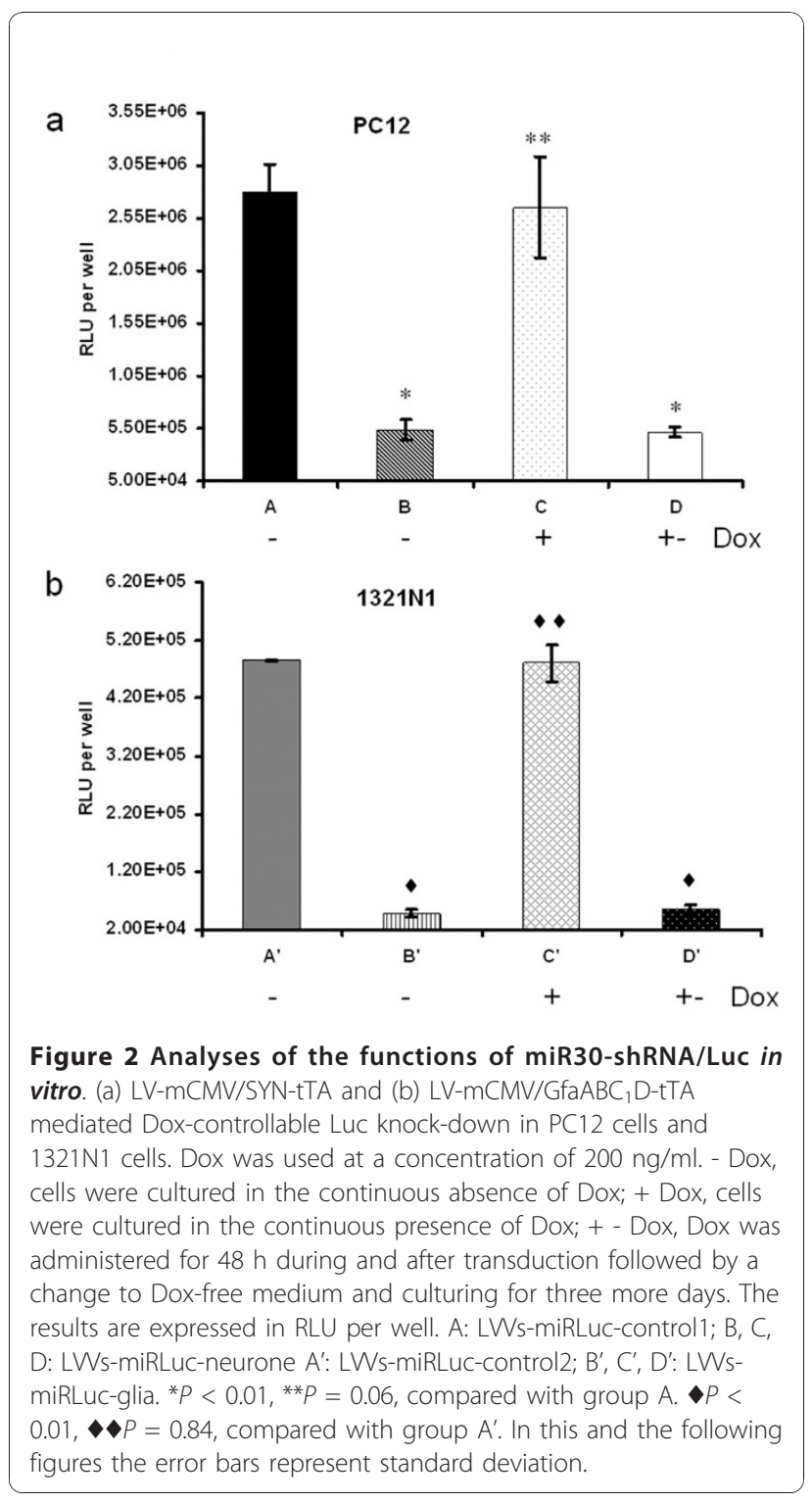

$1321 \mathrm{~N} 1$ cells and LV-mCMV/SYN-tTA in PC12 cells as compared to LV-GfaABC ${ }_{1}$ D-tTA and LV-SYN-tTA respectively. $\mathrm{LV}$-mCMV/GfaABC $\mathrm{m}_{1} \mathrm{D}$-tTA increased tTA expression 14.5 fold as compared to LV-GfaABC ${ }_{1} \mathrm{D}$-tTA while LV-mCMV/SYN-tTA increased 7.6 fold as compared to LV-SYN-tTA (data not shown). Thus, higher efficacy of the astrocytic system is likely to reflect a higher level of tTA in glial cells and therefore a higher expression of the gene knock-down cassette.

\section{Analysis of the effects of miR30-shRNA/Luc in vivo}

Next we investigated the effect of miR30-shRNA/Luc in vivo. LVVs-miRLuc-neurone, LVVs-miRLuc-glia and the corresponding control vectors were injected into the DVC and HIP of adult rats. As shown in Figure $3 a(1)$, in the absence of Dox, LVVs-miRLuc-neurone (Figure 3a, group B) suppressed DVC neuronal Luc expression by $50 \%$ as compared to the level achieved using LVVs-miRLuc-control1 (Figure 3a, group A) $\left(1.85 \times 10^{6} \pm 4.58 \times\right.$ $10^{5}$ vs $3.77 \times 10^{6} \pm 3.70 \times 10^{5}$ RLU per DVC, $\left.P<0.01\right)$. LVVs-miRLuc-glia (Figure 3a, group B') suppressed DVC glial Luc expression even more dramatically (Figure 3a $\sim 85 \%$ ) as compared to level of expression using LVVsmiRLuc-control2 (Figure 3a, group A') $\left(3.69 \times 10^{6} \pm 4.54\right.$ $\times 10^{5}$ vs $2.37 \times 10^{7} \pm 5.71 \times 10^{6}$ RLU per DVC, $P<0.01$ ). This was most likely due to a higher potency of LV$\mathrm{mCMV} / \mathrm{GfaABC}_{1} \mathrm{D}$-tTA as compared to LV-mCMV/ SYN-tTA as revealed by real time PCR analysis of tTA expression from our in vitro work (see previous section). In both cell types, the knock-down could be completely blocked by administration of Dox into the drinking water [group C in Figure 3a(1); group C' in Figure 3a(2)]. We then tested whether gene knock-down in DVC is indeed cell-specific. To do this, we injected the neurone-targeted gene knock-down system (LV-mCMV/SYN-tTA + LVTretight-GFP-miR30-shRNA/Luc) with LVV to express Luc in astrocytes (LV-GfaABC ${ }_{1} \mathrm{D}$-Luc) and, conversely, the astrocyte-targeted knock-down system (LV-mCMV/ GfaABC $_{1}$ D-tTA+ LV-Tretight-GFP-miR30-shRNA/Luc) together with LVV for neuronal Luc expression (LV-SYN-Luc). No knock-down occurred in either case (see Additional file 2) confirming a high degree of cellspecificity of this effect.

Surprisingly, much weaker Luc knock-down was observed using both the neurone-specific and the astrocyte-specific systems, LVVs-miRLuc-neurone and LVVsmiRLuc-glia in HIP compared to DVC [Figure 3b(1), B vs A; Figure $3 \mathrm{~b}(2), \mathrm{B}^{\prime}$ vs A' ]. Reduction in Luc levels with neuronal system was $\sim 27 \%\left(5.5 \times 10^{4} \pm 1.64 \times\right.$ $10^{4}$ vs $7.52 \times 10^{4} \pm 2.48 \times 10^{4}$ RLU per HIP) and $~ 35 \%$ with the astrocytic system $\left(6.32 \times 10^{5} \pm 2.43 \times 10^{5}\right.$ vs $9.65 \times 10^{5} \pm 3.20 \times 10^{5}$ RLU per HIP). Neither effect was significant $(P>0.05)$, although the trend was clear in both cases.

\section{nNOS gene knock-down in vitro and in vivo}

While Luc knock-down is technically convenient it is an artificial approach as this gene is not expressed in the mammalian brain. Thus, it was important to demonstrate whether an endogenous gene is as prone to knock-down as an exogenously expressed transcript. We chose nNOS since this protein is abundantly expressed in both DVC and HIP (Figure 4 and [22-25]).

We first confirmed the efficacy of the anti-nNOS construct, LV-Tretight-GFP-miR30-shRNA/nNOS in PC12 and $1321 \mathrm{~N} 1$ cells. An adenoviral vector (AD) AD-CMV-nNOS was used to induce high level of nNOS expression in both PC12 and 1321N1 cells since there are almost no endogenous nNOS expression in these two cell lines (unpublished observation). As 
a

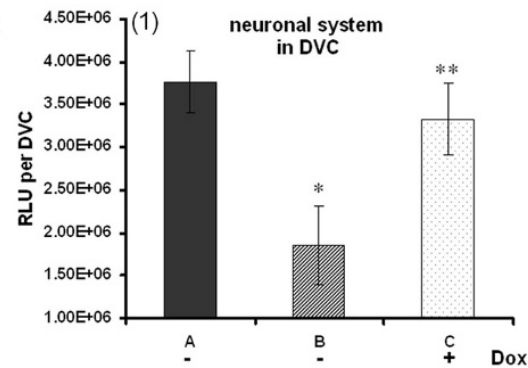

b

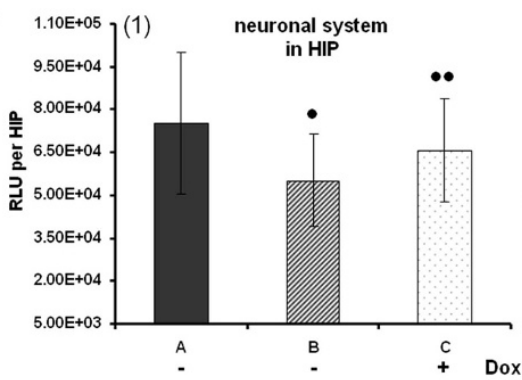

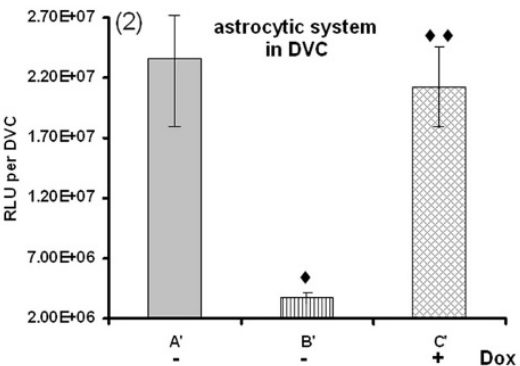

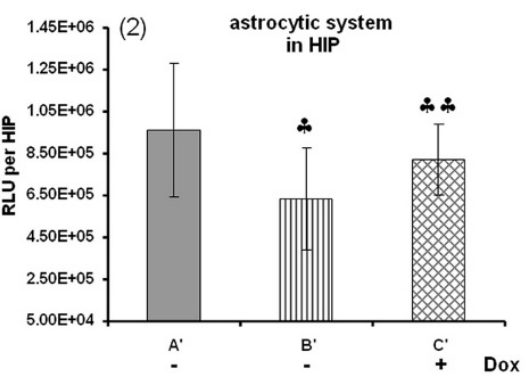

Figure 3 Analyses of the efficacy of miR30-shRNA/Luc in vivo in adult rat brain. a: (1) LV-mCMV/SYN-tTA and (2) LV-mCMV/GfaABC 1 D-tTA mediated Dox-controllable Luc knock-down in DVC in rats. b: (1) LV-mCMV/SYN-tTA and (2) LV-mCMV/GfaABC 1 D-tTA mediated Dox-controllable Luc knock-down in HIP in rats. Rats in groups $A, A^{\prime}, B$ and $B^{\prime}$ were not treated with Dox. Rats in groups $C$ and $C^{\prime}$ drank Dox-containing water post-injection for 7 days. There were 3 rats in each group. A: LWs-miRLuc-control1; B, C: LWs-miRLuc-neurone; A': LWs-miRLuc-control2; $B^{\prime}, C^{\prime}$ : LWs-miRLuc-glia. ${ }^{*} P<0.01,{ }^{* *} P=0.18$, compared with group A. $\bullet P<0.01, \bullet P=0.48$, compared with group $A^{\prime} . . P=0.12, . . P=0.23$, compared with group A. $P=0.03$, $\mathbf{\uparrow} P=0.17$, compared with group $A^{\prime}$.

shown in Figure 4 in the absence of Dox, both LVVsmiRnNOS-neurone and LVVs-miRnNOS-glia markedly knocked down nNOS in PC12 cells $(\sim 69 \%$ reduction in optical density, treatment 2 vs treatment 1 , Figure 4a) and $1321 \mathrm{~N} 1$ ( $~ 82 \%$ reduction, treatment 2 vs treatment 1, Figure 4b). This nNOS knock-down effect could be prevented by Dox (treatment 3 in Figure 4a and Figure $4 \mathrm{~b}$ ). It is important to note that anti-Luc construct, LV-Tretight-GFP-miR30-shRNA/Luc (treatment 4 in Figure 4a and Figure 4b), was without effect in either cell line, indicating that the nNOS knockdown was sequence-specific.
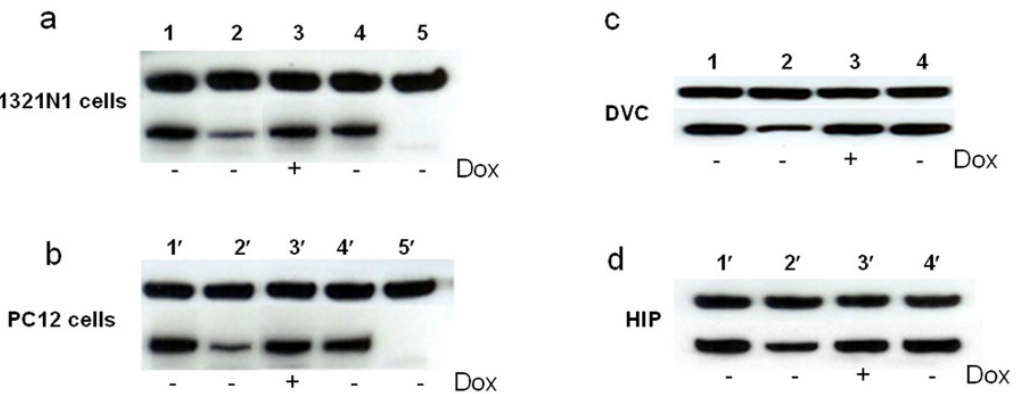

Figure 4 Western-blot analyses of the functions of miR30-shRNA/nNOS both in vitro $(a, b)$ and in vivo (c, d). a: In vitro tests in PC12 cells. - Dox, cells were cultured in the continuous absence of Dox; + Dox, cells were cultured in the continuous presence of Dox; 1: AD-CMV-nNOS + LWs-miRnNOS-control1; 2, 3: AD-CMV-nNOS+ LWs-miRnNOS-neurone; 4: AD-CMV-nNOS+ LWs-miRnNOS-negative control1; 5: mock transfection. b: In vitro tests in 1321N1 cells. 1: AD-CMV-nNOS + LWs-miRnNOS-control2; 2, 3:AD-CMV-nNOS + LWs-miRnNOS-glia; 4: AD-CMV-nNOS + LWsmiRnNOS-negative control2 5': mock transfection. c, d: In vivo tests in DVC (c) and HIP (d) in rats. Rats in groups 2, 4, were not treated with Dox. Rats in group 3 drunk Dox-containing water post-injection for 7 days. There were 3 rats in each group. 1: mock infection; 2, 3: LWs-miRnNOSneurone; 4: LWs-miRnNOS-negative control2. 
A substantial nNOS knock-down ( 55\% reduction) (treatment 2 vs treatment 1, Figure 4c) was observed when LVVs-miRnNOS-neurone was injected into DVC in vivo and consistent with the in vitro data this knockdown could be fully prevented by Dox (treatment 3). However, similar to the experiment with Luc, the nNOS knock-down in HIP ( 35\% reduction; treatment 2 vs treatment 1, Figure 4d) was noticeably weaker. Again, the anti-Luc construct LV-Tretight-GFP-miR30-shRNA/ Luc (treatments 4 in Figure $4 \mathrm{c}$ and Figure $4 \mathrm{~d}$ ) did not trigger nNOS knock-down.

\section{Analysis of miRNA processing enzyme expression in DVC and HIP}

We were surprised to find that the same knock-down cassettes driven by the same targeting systems behaved differently when applied to DVC as compared to HIP. To examine whether the different RNAi efficiency in DVC and HIP is caused by different processing of RNAi, we performed northern blotting analysis to assess the ratio between mature-RNAi and precursor-miR30RNAi in these two regions. We found higher ratio for both Luc RNAi and nNOS RNAi from DVC relative to that from HIP (Figure 5). The ratios of mature RNAi to unprocessed from LVVs-miRLuc-neurone injected rats are $\sim 1: 1$ in HIP and $\sim 18: 1$ in DVC respectively. With LVVs-miRnNOS-neurone, the ratios are $\sim 1.3: 1$ in HIP and $\sim 23.5: 1$ in DVC respectively. We then hypothesized that variations in the composition of the RNAi machinery are a likely cause of this site-specific difference in RNAi processing. To test this idea, we conducted real time RT-PCR analysis of 5 key enzymes implicated in miRNA biogenesis, namely Drosha, DGRC8, Exportin-5, Dicer and Argo (Figure 6a). All five genes tested were found in both DVC and HIP and their expression in

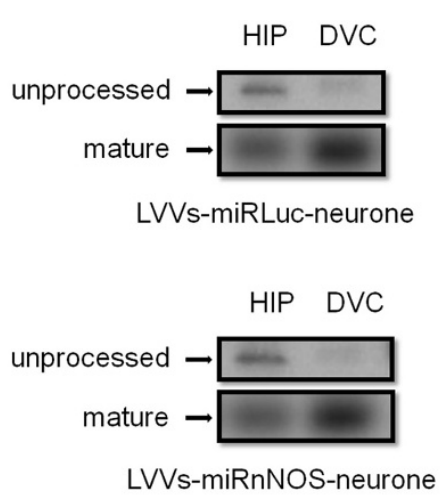

Figure 5 Northern blot analyses to assess the processing of RNAi in HIP and DVC. Total RNA samples were isolated from rats injected with LWs-miRLuc-neurone (upper half) or LWs-miRnNOSneurone (lower half) into HIP and DVC. Blots were probed for either Luc or nNOS shRNA transcripts.
HIP was marginally higher than in DVC. Thus, lower gene knock-down efficacy in HIP is unlikely to be related to regional differences in the availability of the key components on miRNA pathway between DVC and HIP (Figure 6b).

\section{Discussion}

This study was designed to address two questions. Firstly, we wanted to test the efficacy of Pol II-based cell-type specific and Dox-controllable gene knockdown in the brain. Secondly, we wanted to find out whether the same knock-down system should be expected to operate with equal effectiveness in different parts of the CNS. The reason for posing this question is that the lower parts of the CNS, such as medulla oblongata, differ from the higher brain in many respects, including their cellular composition, embryonic origins and gene expression patterns. Neither of these questions has been addressed previously. Moreover, we wanted to assess the effect at the protein level rather than mRNA content since it remains unclear how these two readouts of gene expression are affected by different RNAi constructs, many of which are also thought to operate via translational repression rather than through mRNA degradation.

Lentiviral systems developed in the course of this study enable tight Dox-controllable and cell-specific miR30-based RNAi gene knock-down. Using the Tet system in these designs bring additional benefits because it not only allows switching off the knock-down effect but also simplifies re-targeting of the knock-down vector to different cell types, provided that a sufficiently cell type specific promoter is available. For the Tet system to operate it is essential to achieve high levels of Tet transactivator expression. Mammalian cell-specific promoters are seldom sufficiently powerful, so we have used the previously validated bidirectional TSTA [26] to enhance two such weak promoters here: the SYN and $\mathrm{GfaABC}_{1} \mathrm{D}$. Interestingly, the degree of enhancement achieved using the same bidirectional TSTA strategy was higher for $\mathrm{GfaABC}_{1} \mathrm{D}$ as indicated by a much higher increase in tTA transcription from enhanced $\mathrm{GfaABC}_{1} \mathrm{D}$ compared to enhanced SYN (see results Analyses of the functions of miR30-shRNA/Luc in vitro). This correlated with the overall higher efficacy of gene knock-down achieved with the astrocyte-targeted system both in vitro and in vivo than the neuronal specific system.

Our experiments show that at least in the DVC we can expect a very significant knock-down of the target protein $(\sim 55 \%)$ with our approach. The efficacy of knock-down seems to correlate with the power of the vector used to express the tTA, making astrocytic system slightly more potent than the neuronal one. It is clear that there is no cross-talk between neurone- and 

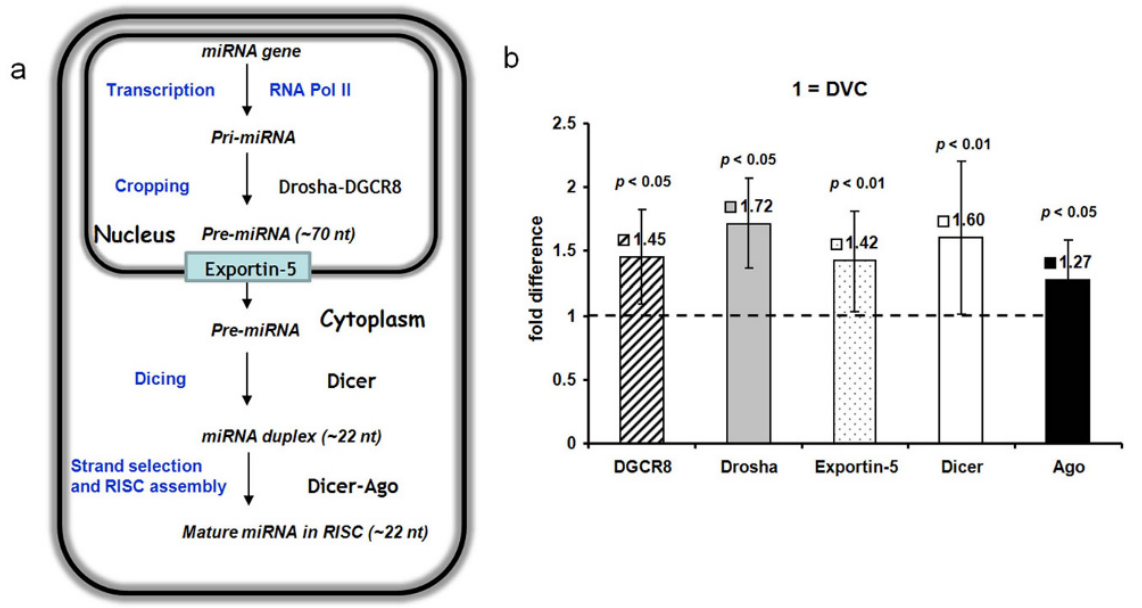

Figure 6 Model for miRNA biogenesis and real time PCR analysis of the five key enzymes implicated in miRNA biogenesis. a: Model for miRNA biogenesis. miRNA genes are transcribed by Pol II to generate the primary transcripts (pri-miRNAs). The initiation step (cropping) is mediated by the Drosha-DGCR8 complex. The product of the nuclear processing is $\sim 70$-nt pre-miRNA. This structure can serve as a signature motif that is recognized by the nuclear export factor Exportin-5. Pre-miRNA constitutes a transport complex together with Exportin-5 and its cofactors. Upon export, the cytoplasmic RNase III Dicer participates in the second processing step (dicing) to produce miRNA duplexes ( 22-nt). The duplex is recognized by the PAZ domain of the Ago protein and incorporated into RISC. Usually one strand is selected as the mature miRNA, whereas the other strand is degraded. $\mathbf{b}$ : Real time PCR analyses of the expression levels of DGCR8, Drosha, Exportin-5, Dicer and Ago in DVC and HIP in rats $(n=5)$. The value for DVC was set as one.

astrocyte-targeted knock-down since when Luc is placed in the phenotype not targeted by the knock-down vectors there is no decrease in expression (see Additional file 2). This also strongly argues against the involvement of any non-specific factors such as local interferon induction. Removal of the knock-down response by Dox (Figures 2, 3 and 4) is another argument for the specific nature of this effect.

Both Pol II and Pol III promoters have been used to express shRNA for RNAi [33]. Unlike most small cellular RNAs, miRNAs are primarily transcribed by Pol II, which may reflect a need for more moderate, regulatable and cell-specific expression. In the context of this study, the Pol II driven production of miRNA-based shRNA allows cell-specific knock-down and exogenous control using the Tet-system. In addition, Pol II promoters in miRNA-based RNAi systems have no strict requirements for the transcriptional start site and termination signal so theoretically various Pol II promoters can be used $[2,9,34]$. Insertion of a GFP reporter gene upstream of miR-shRNAs is thought to have at least two advantages. Firstly, this allows monitoring of the shRNA production in individual cells. Indeed we have noticed GFP expression in our experiments (data not shown). Secondly, the expression of miR-shRNAs and protein mRNAs in a monocistronic transcript could lead to both effective processing of the miR-shRNAs and translation of protein from the mRNA $[15,35,36]$.
It is important to realize that when Luc was used as a target, vectors to express Luc and the knock-down LVVs were applied together as a mixture. While they should have been internalized by the same population of cells in the target area it is possible that some cells would only take in one or two vectors, but not all three. Therefore, a possibility is that some of the residual Luc expression comes from those cells where the binary knock-down system was absent or did not assemble in full (for example, no LVV to express Tre was internalized). When nNOS is chosen as a target, the protein can only come from the cells which endogenously express it in the target area. It is likely that the level of most endogenous genes will be less than those expressed exogenously by a LVV. This could be one of the reasons why nNOS knock-down was evident not only in DVC (as was the case for Luc) but also in HIP (Figure 4c and 4d) although Luc knock-down was only evident as a trend in HIP (Figure $3 \mathrm{~b}$ ).

To our knowledge, this is the first study to evaluate the different efficacies of miR30-based RNAi system among different regions in the brain in vivo. At present we do not know what accounts for this difference. We hypothesized that lower efficacy of the tested constructs in HIP compared to DVC was a result of a lower level of expression of the components involved in the RNAi pathway. However, real-time RT-PCR analysis of Dicer, Argonaute, DGRC8, Exportin 5 and Drosha revealed 
slightly higher levels of all of these transcripts in HIP than in DVC. Hence, other untested or unknown components must account for the difference. RNAi is a dynamic process and its machinery depends on multiple components with only poorly characterized expressions patterns [37]. Moreover, novel components involved in posttranscriptional gene silencing are very likely to emerge. Accumulating evidence indicates that many miRNAs show distinct expression patterns in an organ or tissue-specific way [37-39]. Our constructs, following the design of Stegmeir et al. used flanking and loop sequences from an endogenous miR30 [37]. A recent study uncovered 44 miRNAs which exhibited marked differences in the level of expression between spinal cord, cerebellum and hippocampus in the adult mouse [40]. For example, miR-195, miR-497, and miR-30b were found to be enriched in the cerebellum whereas miR-218, miR-221, miR-222, miR-26a, miR-128a/b, miR-138 and let-7c were highly expressed in the HIP. Unfortunately, the DVC was not studied specifically. It is not impossible that in the brain there are not only regional differences in the levels of expression of the miRNAs but also of some other factors which favour the the production and maturation of specific miRNA. If that was the case, processing of knock-down constructs built using elements of some miRNAs could be more efficient in some parts of the brain than in others as we have found herein. This should be kept in mind for loss-of function studies in the rat brain.

In summary, we have demonstrated that efficient, cellspecific and Dox-controllable gene knock-down can be achieved in the rat brain although the potency of the knock-down using the same construct may differ in different parts of the CNS. This efficient gene silencing system will be a valuable resource for basic gene function study and potentially, for the development of genebased therapeutics of the CNS. While this manuscript was under review, a study where we have used the system for neurone-specific knock-down of nNOS was published [41], demonstrating the practical value of this approach. In that study we demonstrated that nNOS plays a key role in several pathological processes triggered in motor neurones by axotomy. Critically, the knock-down in that experiment was performed in the same brainstem area where, as we show here, this type of RNAi has proven to be most effective. Commercial availability of miRNA-like hairpins might create a feeling that any such construct may be used with the same effect in any part of the brain. This study therefore alerts the readers to avoid such an assumption when planning loss-of function studies in the rodent brain. It also illustrates the fact that more research is needed to perfect and optimise the current strategies for RNAimediated gene knock-down in vivo.

\section{Conclusions}

Selective gene knock-down in subsets of brain cells is achievable; however, there are some presently unknown regional factors which affect either the processing of miRNA-based cassettes or their potency for gene silencing.

\section{Additional material}

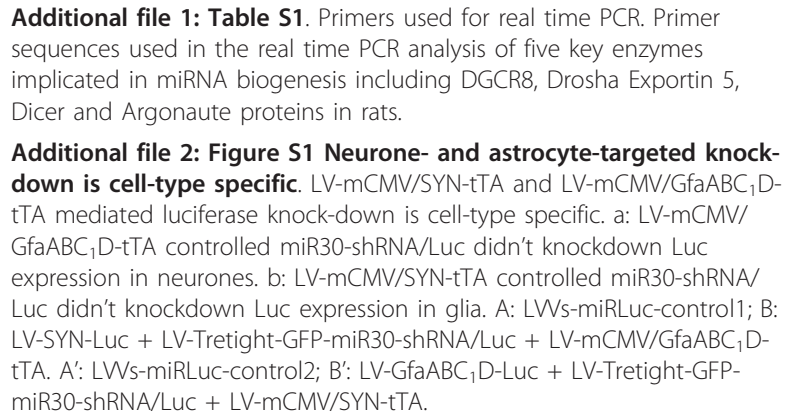

Additional file 2: Figure S1 Neurone- and astrocyte-targeted knockdown is cell-type specific. LV-mCMV/SYN-tTA and LV-mCMV/GfaABC 1 DtTA mediated luciferase knock-down is cell-type specific. a: LV-mCMV/ GfaABC $_{1}$ D-tTA controlled miR30-shRNA/Luc didn't knockdown Luc expression in neurones. b: LV-mCMV/SYN-tTA controlled miR30-shRNA/ Luc didn't knockdown Luc expression in glia. A: LWs-miRLuc-control1; B: LV-SYN-Luc + LV-Tretight-GFP-miR30-shRNA/Luc + LV-mCMV/GfaABC 1 DtTA. A': LWs-miRLuc-control2; B': LV-GfaABC, D-Luc + LV-Tretight-GFPmiR30-shRNA/Luc + LV-mCMV/SYN-tTA.

\section{Acknowledgements}

B Liu, S Kasparov and JFR Paton were supported by the British Heart Foundation RJ/07/006. H Xu was funded by the British Heart Foundation PG/ 06/085. JFR Paton was also in receipt of a Royal Society Wolfson Research Merit Award. The authors thank Samantha Lane and Fiona McBryde for their help in polishing the English of this paper.

\section{Author details}

'Department of Physiology and Pharmacology, Bristol Heart Institute, School of Medical Sciences, University of Bristol, UK. '2Department of Pharmacology, State Key Laboratory for Research and Development of Chinese Materia Medica, Chengdu University of Traditional Chinese Medicine, Chengdu 611137, P.R. China.

\section{Authors' contributions}

$B L$ was responsible for experimental design and completion of the bulk of the laboratory work presented in this article and writing of the manuscript. HX did the western blot analysis. SK designed the study and participated in all aspects of the work. JFRP helped to coordinate the study. All authors have read and approved the final manuscript.

Received: 11 May 2010 Accepted: 6 December 2010 Published: 6 December 2010

\section{References}

1. Dorsett $Y$, Tuschl T: siRNAs: applications in functional genomics and potential as therapeutics. Nat Rev Drug Discov 2004, 3:318-329.

2. Kim DH, Rossi JJ: Strategies for silencing human disease using RNA interference. Nat Rev Genet 2007, 8:173-184

3. Aagaard L, Rossi JJ: RNAi therapeutics: principles, prospects and challenges. Adv Drug Deliv Rev 2007, 59:75-86

4. Soifer HS, Rossi JJ, Saetrom P: MicroRNAs in disease and potential therapeutic applications. Mol Ther 2007, 15:2070-2079.

5. Boudreau RL, Davidson BL: RNAi therapeutics for CNS disorders. Brain Res 2010.

6. Tiemann K, Rossi JJ: RNAi-based therapeutics-current status, challenges and prospects. EMBO Mol Med 2009, 1:142-151.

7. Liu B, Wang S, Brenner M, Paton JF, Kasparov S: Enhancement of cellspecific transgene expression from a Tet-Off regulatory system using a transcriptional amplification strategy in the rat brain. J Gene Med 2008, 10:583-592. 
8. Lonergan $T$, Teschemacher AG, Hwang DY, Kim KS, Pickering AE, Kasparov S: Targeting brain stem centers of cardiovascular control using adenoviral vectors: impact of promoters on transgene expression. Physiol Genomics 2005, 20:165-172.

9. Bartel DP: MicroRNAs: genomics, biogenesis, mechanism, and function. Cell 2004, 116:281-297.

10. McManus MT, Petersen CP, Haines BB, Chen J, Sharp PA: Gene silencing using micro-RNA designed hairpins. RNA 2002, 8:842-850.

11. Zeng Y, Wagner EJ, Cullen BR: Both natural and designed micro RNAs can inhibit the expression of cognate mRNAs when expressed in human cells. Mol Cell 2002, 9:1327-1333.

12. Zeng Y, Yi R, Cullen BR: MicroRNAs and small interfering RNAs can inhibit mRNA expression by similar mechanisms. Proc Natl Acad Sci USA 2003, 100:9779-9784.

13. Dickins RA, Hemann MT, Zilfou JT, Simpson DR, Ibarra I, Hannon GJ, Lowe SW: Probing tumor phenotypes using stable and regulated synthetic microRNA precursors. Nat Genet 2005, 37:1289-1295.

14. Boden D, Pusch O, Silbermann R, Lee F, Tucker L, Ramratnam B: Enhanced gene silencing of HIV-1 specific siRNA using microRNA designed hairpins. Nucleic Acids Res 2004, 32:1154-1158.

15. Stegmeier F, Hu G, Rickles RJ, Hannon GJ, Elledge SJ: A lentiviral microRNA-based system for single-copy polymerase II-regulated RNA interference in mammalian cells. Proc Natl Acad Sci USA 2005, 102:13212-13217.

16. Kafri T, van PH, Gage FH, Verma IM: Lentiviral vectors: regulated gene expression. Mol Ther 2000, 1:516-521

17. Tietge UJ, Kozarsky KF, Donahee MH, Rader DJ: A tetracycline-regulated adenoviral expression system for in vivo delivery of transgenes to lung and liver. J Gene Med 2003, 5:567-575.

18. Schmeisser F, Donohue M, Weir JP: Tetracycline-regulated gene expression in replication-incompetent herpes simplex virus vectors. Hum Gene Ther 2002, 13:2113-2124.

19. Grimm D: Small silencing RNAs: state-of-the-art. Adv Drug Deliv Rev 2009 61:672-703.

20. Murchison EP, Hannon GJ: miRNAs on the move: miRNA biogenesis and the RNAi machinery. Curr Opin Cell Biol 2004, 16:223-229.

21. Boudreau RL, Martins I, Davidson BL: Artificial microRNAs as siRNA shuttles: improved safety as compared to shRNAs in vitro and in vivo. Mol Ther 2009, 17:169-175.

22. Maiti $P$, Singh SB, llavazhagan G: Nitric oxide system is involved in hypobaric hypoxia-induced oxidative stress in rat brain. Acta Histochem 2009.

23. Luo CX, Zhu XJ, Zhou QG, Wang B, Wang W, Cai HH, Sun YJ, Hu M, Jiang J, Hua Y, Han X, Zhu DY: Reduced neuronal nitric oxide synthase is involved in ischemia-induced hippocampal neurogenesis by upregulating inducible nitric oxide synthase expression. J Neurochem 2007, 103:1872-1882

24. Lin LH, Edwards RH, Fremeau RT, Fujiyama F, Kaneko T, Talman WT: Localization of vesicular glutamate transporters and neuronal nitric oxide synthase in rat nucleus tractus solitarii. Neuroscience 2004, 123:247-255.

25. Atkinson L, Batten TF, Corbett EK, Sinfield JK, Deuchars J: Subcellular localization of neuronal nitric oxide synthase in the rat nucleus of the solitary tract in relation to vagal afferent inputs. Neuroscience 2003, 118:115-122

26. Liu B, Paton JF, Kasparov S: Viral vectors based on bidirectional cellspecific mammalian promoters and transcriptional amplification strategy for use in vitro and in vivo. BMC Biotechnol 2008, 8:49.

27. Duale H, Kasparov S, Paton JF, Teschemacher AG: Differences in transductional tropism of adenoviral and lentiviral vectors in the rat brainstem. Exp Physiol 2005, 90:71-78.

28. Coleman JE, Huentelman MJ, Kasparov S, Metcalfe BL, Paton JF, Katovich MJ, Semple-Rowland SL, Raizada MK: Efficient large-scale production and concentration of HIV-1-based lentiviral vectors for use in vivo. Physiol Genomics 2003, 12:221-228.

29. Liu BH, Wang $X, M a$ YX, Wang S: CMV enhancer/human PDGF-beta promoter for neuron-specific transgene expression. Gene Ther 2004, 11:52-60.

30. Chen X, Xu H, Wan C, McCaigue M, Li G: Bioreactor expansion of human adult bone marrow-derived mesenchymal stem cells. Stem Cells 2006, 24:2052-2059
31. Waki H, Liu B, Miyake M, Katahira K, Murphy D, Kasparov S, Paton JF: Junctional adhesion molecule-1 is upregulated in spontaneously hypertensive rats: evidence for a prohypertensive role within the brain stem. Hypertension 2007, 49:1321-1327.

32. Pfaffl MW: A new mathematical model for relative quantification in realtime RT-PCR. Nucleic Acids Res 2001, 29:e45.

33. Wiznerowicz M, Szulc J, Trono D: Tuning silence: conditional systems for RNA interference. Nat Methods 2006, 3:682-688.

34. Yang W, Paschen W: Conditional gene silencing in mammalian cells mediated by a stress-inducible promoter. Biochem Biophys Res Commun 2008, 365:521-527.

35. Shin KJ, Wall EA, Zavzavadjian JR, Santat LA, Liu J, Hwang Jl, Rebres R, Roach T, Seaman W, Simon Ml, Fraser ID: A single lentiviral vector platform for microRNA-based conditional RNA interference and coordinated transgene expression. Proc Natl Acad Sci USA 2006, 103:13759-13764.

36. Dickins RA, McJunkin K, Hernando E, Premsrirut PK, Krizhanovsky V, Burgess DJ, Kim SY, Cordon-Cardo C, Zender L, Hannon GJ, Lowe SW: Tissue-specific and reversible RNA interference in transgenic mice. Nat Genet 2007, 39:914-921.

37. Sood P, Krek A, Zavolan M, Macino G, Rajewsky N: Cell-type-specific signatures of microRNAs on target mRNA expression. Proc Natl Acad Sci USA 2006, 103:2746-2751.

38. Sempere LF, Freemantle S, Pitha-Rowe I, Moss E, Dmitrovsky E, Ambros V: Expression profiling of mammalian microRNAs uncovers a subset of brain-expressed microRNAs with possible roles in murine and human neuronal differentiation. Genome Biol 2004, 5:R13.

39. Oberdoerffer P, Kanellopoulou C, Heissmeyer V, Paeper C, Borowski C, Aifantis I, Rao A, Rajewsky K: Efficiency of RNA interference in the mouse hematopoietic system varies between cell types and developmental stages. Mol Cell Biol 2005, 25:3896-3905.

40. Bak M, Silahtaroglu A, Moller M, Christensen M, Rath MF, Skryabin B, Tommerup N, Kauppinen S: MicroRNA expression in the adult mouse central nervous system. RNA 2008, 14:432-444.

41. Montero F, Sunico CR, Liu B, Paton JF, Kasparov S, Moreno-López B: Transgenic neuronal nitric oxide synthase expression induces axotomylike changes in adult motoneurons. J Physiol 2010, 588:3425-3443.

doi:10.1186/1471-2199-11-93

Cite this article as: Liu et al:: Cell- and region-specific miR30-based gene knock-down with temporal control in the rat brain. BMC Molecular Biology 2010 11:93.

\section{Submit your next manuscript to BioMed Central and take full advantage of:}

- Convenient online submission

- Thorough peer review

- No space constraints or color figure charges

- Immediate publication on acceptance

- Inclusion in PubMed, CAS, Scopus and Google Scholar

- Research which is freely available for redistribution

Submit your manuscript at www.biomedcentral.com/submit
C Biomed Central 